\title{
A Unified Study of Epidemic Routing Protocols and their Enhancements
}

\author{
Zhenxin Feng \\ Informatics faculty \\ University of Wollongong \\ Wollongong, Australia \\ Email: zf984@uowmail.edu.au
}

\author{
Kwan-Wu Chin \\ Informatics faculty \\ University of Wollongong \\ Wollongong, Australia \\ Email: kwanwu@uowmail.edu.au
}

\begin{abstract}
Epidemic protocols belong to a class of routing paradigm that have wide ranging applications in Delay Tolerant Networks (DTNs) due to their simplicity, low delays, and little to no reliance on special nodes. To this end, a comprehensive study of their performance will serve as an important guide to future protocol designers. Unfortunately, to date, there is no work that studies epidemic routing protocols using a common framework that evaluates their performance objectively using the same mobility model and parameters. To this end, we study four categories of epidemic routing protocols. Namely, P-Q epidemic, epidemic with Time-To-Live (TTL), epidemic with Encounter Count (EC) and epidemic with immunity table. Our results show that the probability of transmissions as used in P-Q epidemic may increase delay and decrease delivery ratio. Apart from that, an incorrect TTL value leads to premature discarding of bundles, and thereby, has a non negligible impact on delivery ratio. Epidemic with EC suffers from high buffer occupancy levels and long delivery delays. In addition, epidemic with immunity suffers from high overheads. Henceforth, we propose three enhancements: dynamic TTL, EC+TTL and cumulative immunity to address the aforementioned limitations. Our results show that dynamic TTL improves delivery ratio by more than $20 \%$, EC+TTL reduces buffer occupancy level by $40 \%$, and improve delivery ratio by at least $40 \%$ at high loads. Cumulative immunity reduces the buffer occupancy level of nodes by at least $15 \%$ whilst incuring an order of magnitude less signaling overheads.
\end{abstract}

Index Terms-routing protocol; delay tolerant networks; epidemic; performance evaluation;

\section{INTRODUCTION}

Delay Tolerant Networks (DTNs) are characterized by large delays, frequent disruptions and lack of contemporaneous paths between nodes. In addition, nodes may have limited computational power, storage, and battery capacity. Despite these challenging conditions, nodes are able to transmit messages, and the resulting network has proven to be applicable in a variety of scenarios. For example, in ZebraNet [1], nodes correspond to wild zebras in a national park. Each zebra has a sensor that tracks and collects its movements and location. The resulting sensed data is then collected by researchers. Interestingly, in [2], the authors showed that sending a 32 Gigabytes message in the same city using a pigeon to have higher bandwidth and shorter delivery time than transmitting the same message via the Internet.

A key problem in DTNs is designing routing protocols that deal with the following key challenges and issues: stochastic and dynamic topologies, limited topology information, variable and uncertain contacts between nodes. For example, the movement of mobile nodes or students in Fig.1 is uncontrolled, and exhibits varying temporal and spatial characteristics. More importantly, they do not have any topological information which they can use to route a packet efficiently. A challenging issue here is that nodes have independent movement patterns. Hence, nodes experience variable and uncertain rendezvous periods and frequencies. Note, from here on, we will use the term "bundle" to denote messages in DTNs.

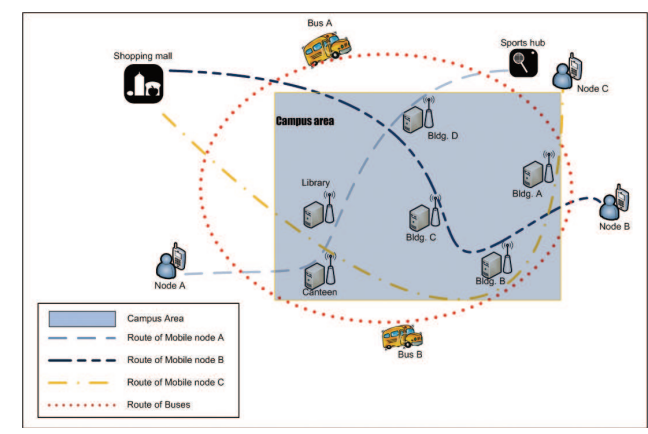

Fig. 1. An example DTN operating on a university campus.

To date, there are three types of DTNs routing protocols: (i) epidemic (ii) Data Ferry (DF) and (iii) statistical. In type (i), nodes cooperatively route bundles for their neighbors. For example, in Fig.1, given mobile node A, B and C are likely to encounter each other at some point on their respective path, node $\mathrm{C}$ can help transmit bundles from node $\mathrm{A}$ to $\mathrm{B}$. That is, node $\mathrm{A}$ transmits its bundles for node $\mathrm{B}$ via node- $\mathrm{C}$. This means, once node $C$ receives the bundle from node $A$, it sends them to $\mathrm{B}$ at a later rendezvous time. Routing protocols of type (ii) take advantage of mobile nodes called data ferries. Lastly, routing protocols of type (iii) utilize statistical methods to avoid arbitrary flooding. The key assumption is that nodes in a DTN will always encounter one another. Hence, each node can compile a set of statistics or metrics regarding their mobility pattern. Other metrics include the number of times a node has encountered a given node, and the duration in which a node remains connected with a given neighbor. 
This paper focuses on epidemic routing protocols. They are ideal for use in a variety of DTNs. For example, those based on the random mobility of humans [3][4]. This is because they have a simple bundle transmission procedure that only relies on nodes encounters. Moreover, they do not assume the existence of special nodes. In particular, they do not rely on nodes with ample resources or pre-determined movement patterns. These properties are particular suited for DTNs that use resource constrained sensor nodes; e.g., [5]. Epidemic routing protocols are also critical to one-to-all communication schemes, which can be used to disseminate advertisements or events [6] [7]. More importantly, according to [8], epidemic routing protocols are able to achieve minimum delivery delay, but at the expense of higher resource usage - a key issue addressed in this paper.

Another important issue addressed in this paper is the lack of research that evaluates epidemic routing protocols using a unified framework. In particular, epidemic routing protocols have been tested in different scenarios in terms of number of nodes, network area, buffer size and bundle size; see Table I. As a result, it is very difficult to compare epidemic routing protocols objectively. Besides that, no work has compared the performance of epidemic routing protocols using both the Random Way Point (RWP) model [9] and trace files.

TABLE I

EXPERIMENT PARAMETERS USED IN STUDIES SUCH AS [10][11][12][13].

\begin{tabular}{|l|l|}
\hline Number of Nodes & $\leq 100$ \\
\hline Mobility Pattern & Random Waypoint \\
\hline Network Area & $\leq 50 \mathrm{~km}^{2}$ \\
\hline Transmission Range & $\leq 300 \mathrm{~m}$ \\
\hline Metircs & $\begin{array}{l}\text { Delivery ratio, average delay, } \\
\text { time to deliver all bundles }\end{array}$ \\
\hline Buffer Size & Infinite or up to 5 MB \\
\hline Bundle Size & $\leq 14 \mathrm{MB}$ \\
\hline
\end{tabular}

Henceforth, we aim to shed light on the performance of epidemic routing protocols under a unified framework. Specifically, we compare all epidemic routing protocols using a custom-built simulator that moves nodes according to a trace-file and the RWP model. Moreover, we compare these protocols using the same set of parameters; e.g., node numbers, load and buffer space. From our extensive simulation studies, we identified the following limitations with existing epidemic based routing protocols: high buffer occupancy level, premature discard of bundles, inefficient use of immunity tables to purge redundant bundles, low delivery ratio at high loads, and poor adaptivity to changing network parameters.

This paper also contains three key enhancements to address the aforementioned limitations. First, we set the Time to Live (TTL) parameter of bundles dynamically according to a node's encounter interval. The intuition here is that bundles should be buffered according to the interval between two encounters. That is, when nodes experience a long inter-contact interval, bundles will have a larger TTL value, whilst a short interval results in small TTL value. Our results show epidemic with dynamic TTL improves delivery ratio by more than $20 \%$. Second, we combined Encounter Count (EC) with TTL to reduce buffer occupancy level and increase bundle delivery ratio. The resulting combination is able to reduce buffer occupancy level by $40 \%$. More importantly, it dramatically improves the delivery ratio by at least $40 \%$ at high loads. Third, we improve the use of immunity tables to carry a cumulative acknowledgment. This has the effect of facilitating buffer discard policy, and more importantly, allows a node to delete multiple bundles upon receiving one immunity table. This is an improvement over past studies as nodes need to receive $N$ immunity tables in order to delete $N$ bundles. We have conducted extensive experiments using both the RWP model and trace-file to verify these enhancements, and our results confirm their superiority over existing epidemic routing protocols.

The rest of this paper is organized as follows. Section II presents an overview of epidemic routing protocols, their taxonomy and shortcomings. Section III describes our enhancements to each epidemic routing protocol. Section IV presents our research methodology and Section $\mathrm{V}$ shows our experiments results. Section V-C and VI include our discussion and conclusion respectively.

\section{BACKGROUND}

\section{A. Pure Epidemic}

Vahdat et al.[13] proposed the first epidemic routing protocol, called pure epidemic from here on, for DTNs, in which nodes transmit new bundles to a peer in each encounter. Each node records received bundles using a summary vector, which it then uses to determine "new" bundles that are to be exchanged in the current encounter. This comparison, so called anti-entropy session, ensures nodes only exchange missing bundles. As shown in Fig.2, node A and B exchange bundles which are different to those in their buffer. The downside of pure epidemic is that the buffer occupancy of each node rises with each encounter. Hence, it needs an appropriate buffer management policy that frees up buffer space whilst ensuring high bundle delivery ratio.

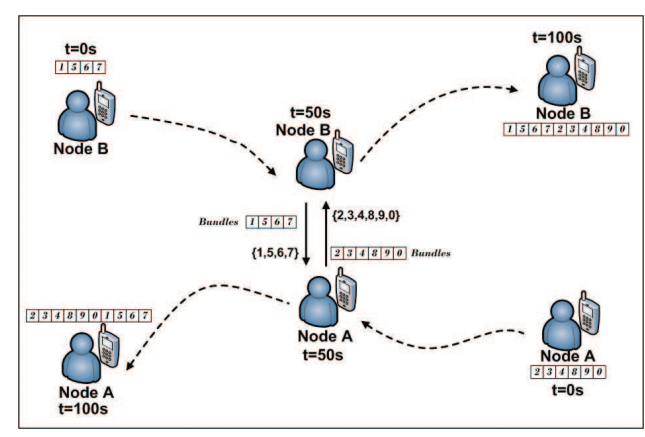

Fig. 2. Pure epidemic transmission.

\section{B. Optimizations}

There are two main approaches to address the high buffer occupancy level of pure epidemic routing protocol: antipackets and metrics. Anti-packets are generated by a destination node when it has received a bundle. The resulting 
anti-packet is then used by nodes in the network to deliver the corresponding bundle. In other words, each bundle is paired with an anti-packet, and the relationship is analogous to "infection and vaccination" in epidemiology. In Fig.3, nodes first exchange anti-packets. Accordingly, node A determines that bundle 2, 3 and 4 have been delivered to their destination. Therefore, node A deletes them from its buffer and only deliver bundle $8,9,0$ to node $\mathrm{B}$. The transmission from node $\mathrm{B}$ to A follows the same process. Compared to pure epidemic in Fig.2, nodes with immunity tables are able to reduce their buffer occupancy level from 10 bundles to five bundles; see Figure 2 and 3. Moreover, the use of anti-packets, which are usually small in size, reduce the number of bundles that are exchanged in each encounter. Example protocols that employ this optimization include epidemic with immunity [12] and P-Q epidemic [10].

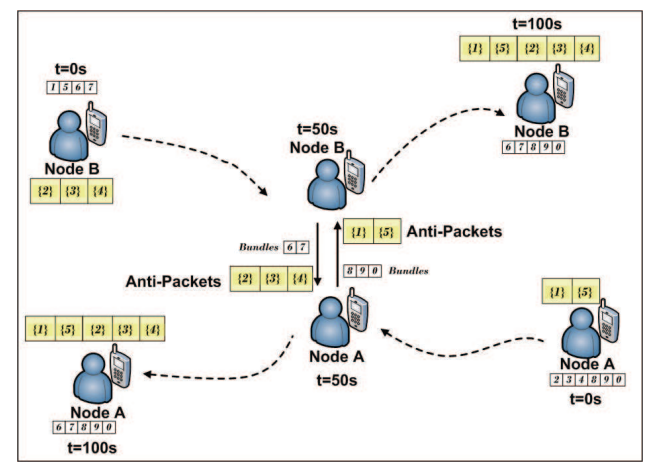

Fig. 3. Epidemic with anti-packets.

In epidemic with immunity[12], nodes deliver and drop bundles according to two lists: $\mathrm{m}$-list and i-list. The $\mathrm{m}$-list is similar to the summary bundles vector in pure epidemic, which records received bundles. More importantly, i-list is updated whenever nodes receive an immunity table, where it specifies bundles that have arrived at their respective destination. When two nodes encounter each other, they combine their immunity tables into one i-list, check each other's buffer and delete redundant bundles according to this i-list.

In P-Q epidemic[10], nodes not only reduce their buffer consumption by using anti-packets, nodes also employ a probabilistic transmission process to exchange bundles. In particular, a source node sends bundles according to probability $\mathrm{P}$, while other nodes transmit their bundles with probability Q. Moreover, anti-packets are disseminated when bundles are received by their destination. Fig. 4 illustrates P-Q epidemic in operation. When node $A$ and $B$ encounter each other, they transmit their bundles according to $\mathrm{P}$ and $\mathrm{Q}$ value, which are set to 0.5 in this example.

In another optimization, nodes compute a metric that reflects the current network state, such as the number of times a node has encountered another node or contact duration in order to evaluate a neighbour's ability to deliver a bundle successfully. Examples include epidemic with EC [14] and epidemic with TTL [15].
In [14], each node discards bundles according to their EC, which is increased by one whenever a bundle is transmitted to another node. Hence, a high EC means there are many duplicates in the network, and thus can be safely overwritten by new bundles. Fig. 5 shows how bundles with the highest EC value are replaced by newly received bundles. Each bundle has an attached EC value which is stored in the EC table. Once bundles are exchanged, their EC value is increased by one. In the figure, node A transmits bundle 4,8 and 9 to node $\mathrm{B}$, which results in them having a new EC value of 4,3 and 7 respectively. Given that node A and B's buffer is only capable of storing five bundles, when node B's buffer is full, bundle 3 and 6 are discarded and replaced by bundle 8 and 9 as they have the highest EC value. Note, undelivered bundles have higher priority even though they have a higher EC value. For example, in Fig.5, because node B has never received bundle 9 , node B replaces bundle 6 with bundle 9 , which has a higher EC value.

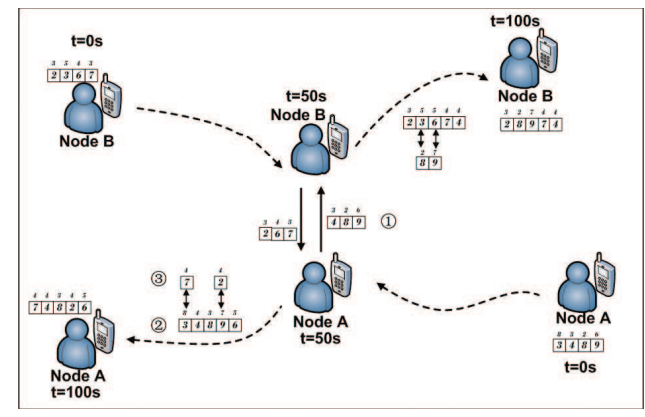

Fig. 4. Epidemic with EC.

In Epidemic with TTL [15], nodes discard bundles according to bundles' TTL value. Every bundle has the same TTL, and once they are transmitted and stored in a buffer, their TTL begins to reduce by one every second. If a bundle is transmitted to other nodes before its TTL expires, the bundle's TTL value is renewed. As shown in Fig.6, the bundles stored in node A and $\mathrm{B}$ are removed after $\mathrm{t}=50 \mathrm{~s}$ as both nodes fail to forward these bundles to another node.

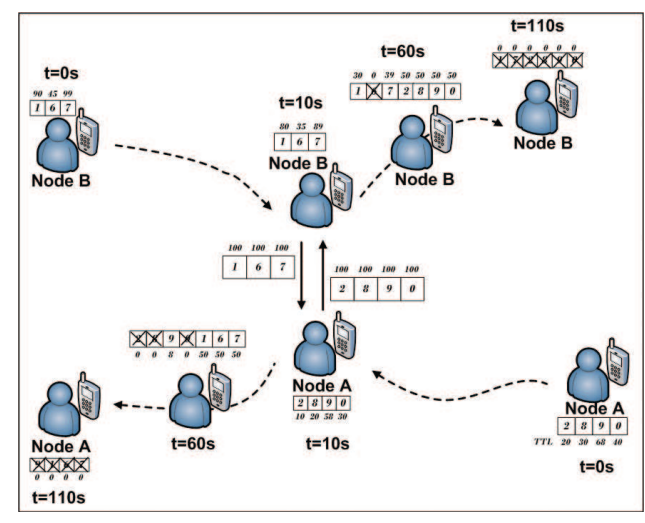

Fig. 5. Epidemic with TTL. 


\section{Discussion}

In $\mathrm{P}-\mathrm{Q}$ epidemic, setting $\mathrm{P}$ and $\mathrm{Q}$ to be less than one may increase transmission delay and decrease delivery ratio. Specifically, in an encounter, assume two nodes can transmit up to 10 bundles. If $\mathrm{P}=\mathrm{Q}=0.5$, neither nodes can transmit all 10 bundles. This means they are not fully taking advantage of their encounter. This also means nodes are required to encounter each other more often in order to deliver bundles. Unfortunately, in DTNs, nodes are not guaranteed to encounter each other frequently. Every encounter is important, and a missed opportunity will likely result in long delays and low delivery ratio.

We observe that epidemic with fixed TTL values to be poorly suited for use in DTNs. This is because setting a large TTL value can result in nodes storing bundles that have arrived at their respective destination. On the other hand, small TTL values lead to bundles being discarded prematurely. This is especially critical if nodes are discarding bundles that have a low duplication rate as doing so leads to transmission failure. The primary problem in epidemic with EC is that nodes experience high buffer occupancy levels and longer delivery delays than other protocols; see Section V. This is because nodes delete their bundles that have the highest EC value from their buffer only when it is full. Moreover, discarding bundles before they are received by the destination reduces bundle delivery ratio.

In epidemic with immunity, a destination node generates an immunity table whenever it receives a bundle successfully. That is, each immunity table identifies one bundle. Hence, the number of immunity tables transmitted is proportional to the load. As a result, they may cause congestion and consequently, result in the discard of bundles. This is particularly detrimental if the discarded bundles have not been forwarded to other nodes.

\section{ENHANCEMENTS}

In this section, we propose three enhancements that address the limitations presented in Section II-C.

- Epidemic with TTL. To prevent bundles from being discarded prematurely or buffered unnecessarily due to improper TTL values, we set the value of TTL dynamically; see Algo-1. More specifically, a bundle's TTL value is set to double the interval time between the last two encounters. This means longer interval results in larger TTL values, and vice-versa. The intuition here is that a longer interval means a DTN is sparse, and hence, bundles should be buffered for a longer period of time to ensure successful delivery.

- Epidemic with EC. We define a minimum EC value before nodes are allowed to delete a bundle; see Algo. 2. In addition, when the EC value of bundles exceeds a given threshold value, bundles will be given a TTL value. The TTL value will depend on a bundle's EC values. In particular, the TTL of a bundle is proportional to the number of times it has been transmitted. In our

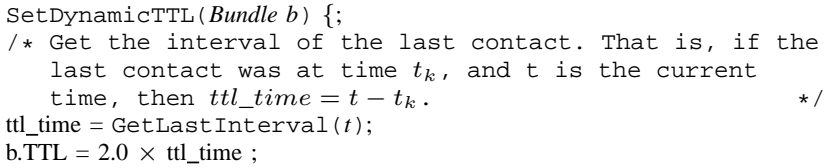

Algorithm 1: Pseudo-code used to set the TTL value of each bundle

experiments, when bundles are transmitted over eight times, bundles will be given a TTL value of 300 . For each additional transmission, their TTL value will be reduced by 100 seconds.

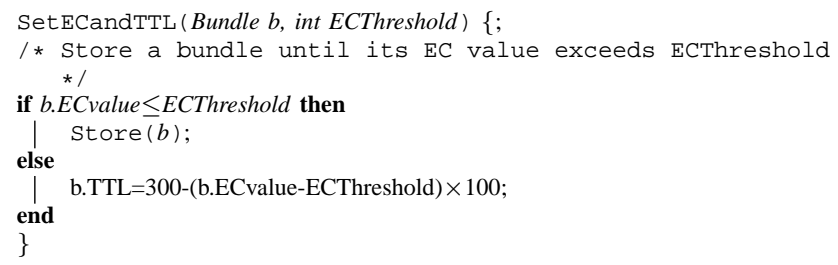

Algorithm 2: Pseudo-code used to set the EC and TTL value of each bundle

- Epidemic with Immunity. We introduce a cumulative immunity table; see Figure 7. For example, an immunity table with a bundle ID of 30 means the destination node has received bundles 1 to 30. Note that, destination generates a cumulative immunity table only when it has received one or more bundles successfully. The destination transmits an immunity table for each node that it meets. In terms of buffer policy, a node removes any immunity tables that are redundant. That means, if there are two immunity tables that cover bundles with ID up to 30 and 50, the node will delete immunity table that covers the first 30 bundles.

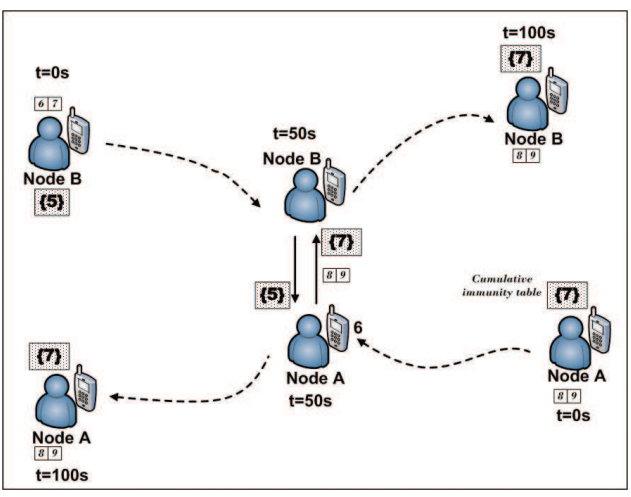

Fig. 6. Epidemic with cumulative immunity table.

\section{Research Methodology}

Our experiments are based on the data collected by Scott et al. [16]. They collected data over a five day period for the following scenario. Students were asked to carry short range 
communication devices at the University of Cambridge. In total, there are 12 devices. Each device has a unique ID and records the following information for every node it encounters: begin times, duration and number of encounters. The data reflects students' rendezvous behaviours. In particular, the trace file shows nodes/students are not always connected, and hence they experience large delays between meetings. Secondly, nodes' movement and encounter duration are random.

We have developed a custom simulator to conduct our experiments. The simulator models bundles exchanges and the mobility of nodes. In particular, we have implemented all the epidemic routing protocols outlined in Section II. It takes as input a trace-file that describes the mobility of nodes; notably, it works on traces available from CRAWDAD [17], which allows us to experiment with realistic mobility patterns.

At the start of each simulation, the trace file is processed event by event. The transmission of bundles begins and finishes at the start and end of each node encounter respectively. Bundles are generally much bigger than messages in conventional networks. For example, bundles in [18] range from several hundreds of Megabytes to Terabytes. Consequently, in our experiments, we fix the transmission time to 100 seconds. For example, event \#1 and \#2 record indicate that Node 3 and 9 have a encounter duration of $3882-3568=314$ seconds, and in this duration, Node 3 sends [314/100] =3 bundles to Node 9.

Using the said datasets, we performed a trace-based simulation study of different DTN routing protocols. We set each node to hold 10 bundles. The transmission rate, which is the number of bundles that can be transmitted in one time unit, is set to 1 bundle/second. This means the number of bundles exchanged is directly proportional to the rendezvous duration. In each transmission, there is only one source and destination node.

In our experiment, a source node is chosen randomly, and transmits $k$ bundles to a destination node. We increase the value of $k$ by five after each experiment, and set the maximum number of bundles to 50. For each $k$ value, we run the simulation 10 times and average our results; note, additional simulation runs did not yield any discernible changes in our results. We also change the source and destination node after each run. Moreover, to avoid collision, the node with the lower ID will send first. Once the destination received all bundles, the simulation ends. Also, the maximum recorded time from the trace file is $524,162 \mathrm{~s}$. This means if the simulation exceeds this time, the destination node may not have a chance to receive all bundles. In this case, we mark the transmission as failed, and no delays will be recorded.

We also study and evaluate each protocol when nodes move according to the Random Way Point (RWP) model [9]. Specifically, we simulated 12 nodes moving according to the RWP model within a 600,000 seconds period. Nodes randomly choose a destination point, and moves at a speed ranging from 0 to $200 \mathrm{~m} / \mathrm{s}$. Nodes may be in contact, whilst on the move or stationary, for a maximum 500 seconds. In both scenarios, once nodes encounter each other, they begin to exchange bundles.

We note that RWP has two fundamental problems [19]. First, any experiments employing RWP may result in nodes having odd movements such as circular or zig-zag patterns. Second, an improper velocity value can lead to all nodes becoming stationary after some period of time. To avoid these problems, we generate a RWP trace-file that ensures nodes move continuously along rendezvous points until the end of the simulation. Specifically, there are less than 100 subscriber points in a one square kilometre area, and nodes encounter and exchange bundles at each point. When nodes reach one subscriber point, they will randomly stop for less than 1000 seconds and move to the next subscriber point, which is also chosen randomly. Note that, the speed that nodes move from one subscriber point to another is dependent on the distance between points, and the interval between contacts, as recorded in the trace file. The speed is then calculated as distance/interval time. In our experiments, the distance between any two subscriber points is less than 1000 meters. In our experiments, the maximum distance between any two subscriber points is 1,000 meter, and the minimum interval time is 100 seconds, therefore, the velocity of nodes in our experiments ranges from 0 to $10 \mathrm{~m} / \mathrm{s}$. Note that, 0 is the speed when nodes encounter and exchange bundles at subscriber points.

In experiments where nodes use pure epidemic, they transmit bundles according to their encounter duration as determined by the trace file. More specifically, if two nodes meet each other, the number of bundles that will be transmitted is dependent on nodes' transmission rate and their encounter duration. In P-Q epidemic, recall that a source node sends bundles according to probability $\mathrm{P}$, while other nodes transmit their bundles as per probability $\mathrm{Q}$. We experiment with the following $\mathrm{P}$ and $\mathrm{Q}$ values: $0.1,0.5$ and 1 . In epidemic with TTL, we experimented with TTL values of 50, 100, 150 and 200 seconds. In all our experiments, we recorded the following metrics:

- Buffer occupancy level - the average buffer utilization of all nodes.

- Bundle duplication rate - the number of nodes in the network that has a copy of a given bundle over the total number of nodes in the network. For example, a bundle duplication rate of $50 \%$ means half of the nodes in the network have a copy of a given bundle.

- Delivery ratio - a metric that reflects how many bundles have been delivered successfully to their destination. More specifically, the ratio of received bundles over the total number of bundles sent by the source.

- Delay - the time taken for all bundles to arrive at their respective destination.

\section{Results}

In the following section, we first compare existing epidemic routing protocols using both RWP and trace-file simulation. As we pointed out in Section II-C, such comparison has never been carried out in past studies. From our experiments, we 
highlight the key limitations of each epidemic routing protocol, which serve to justify the enhancements proposed in Section III. After that, in Section V-C, we evaluate the effectiveness of our enhancements in addressing these limitations.

\section{A. Existing Epidemic-Based Protocols}

The delay experienced by epidemic routing protocols are reported in Fig. 7 and 8. We use the parameters that result in the best delay for all protocols. For P-Q epidemic, we chose $\mathrm{P}=\mathrm{Q}=1$, and for epidemic with TTL, we set the TTL to 300 seconds. Note that, because P-Q epidemic and epidemic with immunity have the same delay in trace-based experiments when $\mathrm{P}=\mathrm{Q}=1$, we only plot the delay curve of $\mathrm{P}-\mathrm{Q}$ epidemic in Fig. 7. We can see that with increasing load, the delay of epidemic with EC grows the quickest, and P-Q epidemic has the slowest growth. The reason is because epidemic with $\mathrm{EC}$ is able to delete bundles before they are received by their destination, which leads to higher delay. Furthermore, in Fig. 8 , we can see that epidemic with TTL has a higher delay than epidemic with immunity. This is because the value of TTL is fixed, whilst epidemic with immunity discard bundles as soon as nodes receive an immunity table. A key observation is that nodes frequently delete bundles as the TTL value of bundles is shorter than their encounter interval. As a result, the network will have fewer duplicated bundles, and consequently, have a low bundle delivery ratio because destination nodes are less likely to meet nodes with the required bundles.

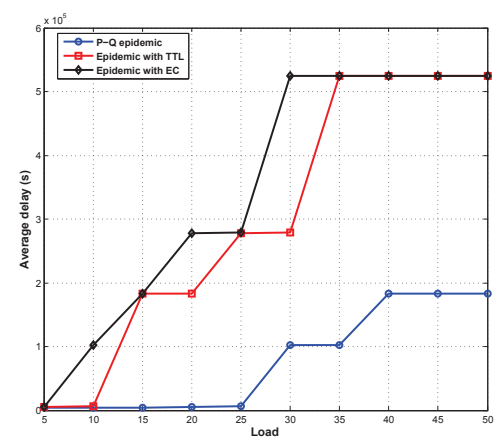

Fig. 7. Delay comparison of epidemic-based protocols when nodes move according to the trace file.

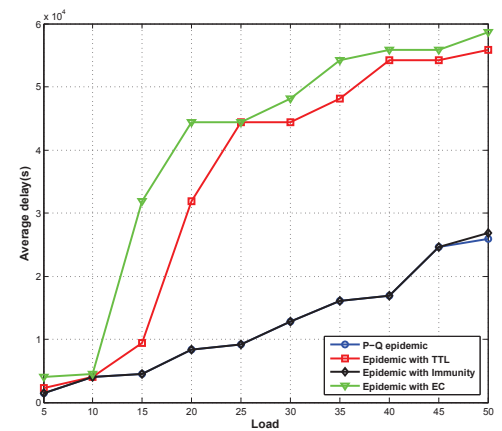

Fig. 8. Delay comparison of epidemic-based protocols under RWP.
Fig. 9 and 10 show epidemic with EC has a lower bundle duplication rate than other protocols. However, epidemic with EC fares better as it discards bundles frequently, which unfortunately lowers bundle delivery ratio. Moreover, epidemic with immunity has a high bundle duplication rate, which reaches over $60 \%$. This is due to the following reasons. First, dissemination of immunity tables relies on the frequency of nodes encounters, and the number of immunity tables, which is equal to the number of bundles or load. Our results show that immunity tables are propagated slowly. As a result, nodes that have not received any immunity tables will continue to transmit the corresponding bundles. Second, when nodes free up their buffer, they are able to store more undelivered bundles and exchange them when they encounter each other. Consequently, freeing up buffer space also plays a role in increasing duplication rate. Apart from that, P-Q epidemic also has a high bundle duplication rate. This is because when $\mathrm{P}=\mathrm{Q}=1, \mathrm{P}-\mathrm{Q}$ epidemic is similar to pure epidemic. In other words, the bundle duplication rate is proportional to nodes' encounter frequency.

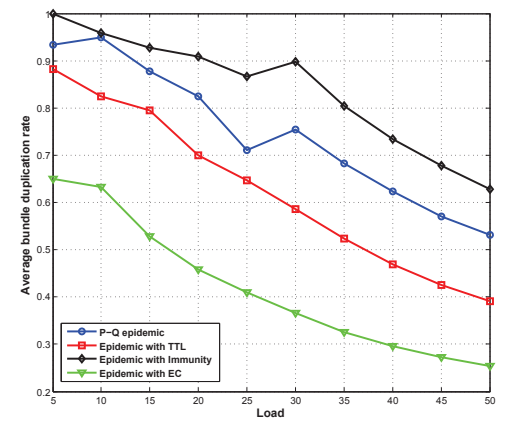

Fig. 9. Average bundle duplication rate of epidemic-based protocols when nodes move according to the trace file.

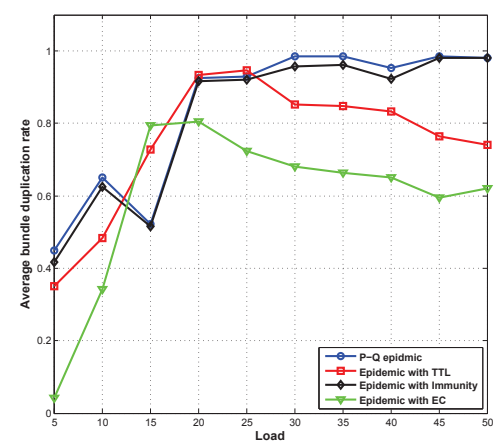

Fig. 10. Average bundle duplication rate comparison of epidemic-based protocols when nodes move according to the RWP model.

From Fig. 11 and 12, we see that P-Q epidemic consume more than $80 \%$ of nodes buffer when the load is higher than 10 in both trace-based and RWP studies. This is because after bundles are received by the destination, the protocol does not have any mechanism to purge these bundles. Other protocols, however, have such a mechanism. For example, epidemic with immunity table discards transmitted bundles according to its 
immunity table, which results in a $10 \%$ decrease in buffer occupancy level. Furthermore, because epidemic with TTL discards bundles before they are received by their respective destination, its low buffer occupancy level has a negative effect on transmission. Note, when the load is more than 20 , the buffer occupancy level of epidemic with immunity experience sudden drops and rises in both trace file and RWP model. This is because epidemic with immunity only discards bundles after they reached their respective destination. Therefore, nodes' buffer occupancy is dependent on immunity tables stored in each node.

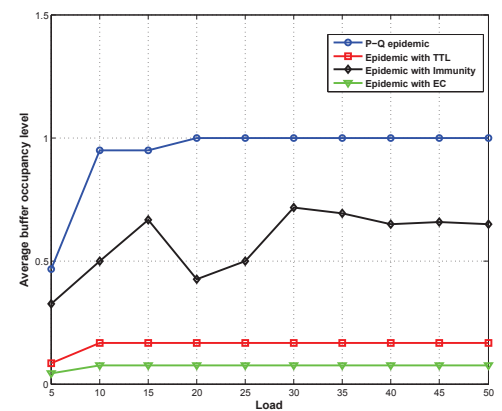

Fig. 11. Buffer occupancy level comparison of epidemic-based protocols.

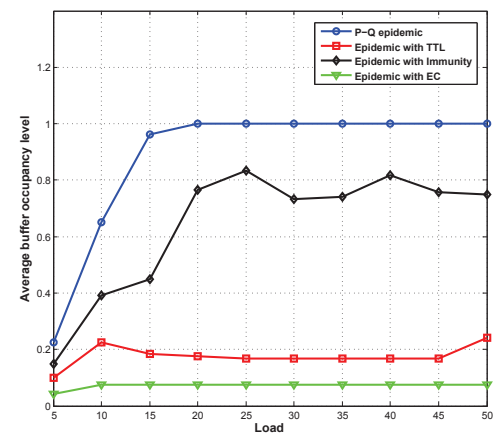

Fig. 12. Average buffer occupancy level comparison of epidemic-based protocols in RWP studies.

In our trace-based experiments, we only compare the delivery ratio of epidemic with EC and TTL because other protocols have a $100 \%$ delivery ratio. As shown in Fig. 13, when the load increases, the delivery ratio of all protocols reduces accordingly. We observe that TTL is not suitable for use in DTNs because of the following reason. Nodes experience large encounter intervals, much more than the delays experienced by nodes in conventional networks. In practice, each network will have specific encounter characteristics, where nodes rendezvous interval may range from a few seconds to days. Consequently, delays may be unbounded, and hence, it is challenging to select a TTL value that can be used to safely discard bundles.

Our experiments yield the following findings: first, a high duplication rate leads to short delays. For example, epidemic with immunity has a shorter delay and higher bundle duplication rate than other protocols, whilst epidemic with EC

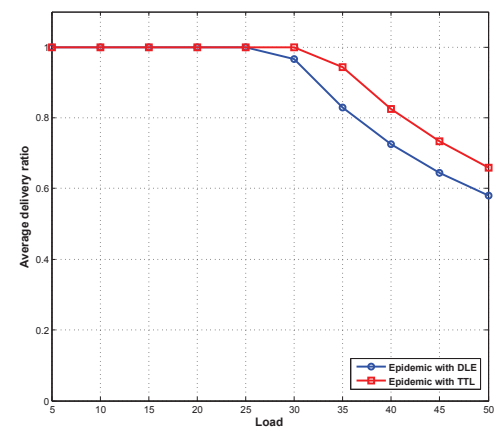

Fig. 13. delivery ratio comparison of epidemic with TTL and EC.

has a longer delay and a lower bundle duplication rate as compared to other protocols. This is because as more nodes have the required bundles, more bundles will be delivered to their respective destination. Second, deleting bundles that are enroute to their destination may result in the deletion of bundles that have a low duplication rate, which unfortunately leads to increased delay or low delivery ratio. Ideally, if we know which bundles have arrived safely, then any duplicates can be deleted without sacrificing delay or delivery ratio. This helps reduce buffer occupancy level, and enable nodes to store bundles that have not reached their destination. Unfortunately, the propagation of feedback generated by a destination is also governed by the contact characteristics of nodes.

\section{B. Enhanced Protocols}

We now focus on the enhancements presented in Section III. Again, we use both the RWP and trace-file in our experiments. We compare protocols with our enhancements against their corresponding un-modified version. Additionally, in all scenarios that deploy epidemic with constant TTL, we set the TTL value, which is constant, to 300 seconds.

1) Delivery Ratio: We first show that encounter interval time has a significant effect on the delivery ratio of epidemic with constant TTL. For example, we deployed two network scenarios to evaluate the influence of encounter interval on epidemic with TTL. Both scenarios include 20 nodes, each of which has at most 20 encounters with other nodes. The only difference between these two scenarios is that the interval time between two successive encounters is set to a maximum of 400 and 2000 seconds respectively.

Fig. 14 shows the delivery ratio achieved by epidemic with constant TTL value. We see that when the interval between encounter increases, delivery ratio drops dramatically - viz. an interval time of 2000 has a $20 \%$ lower delivery ratio than an interval time of 400 . The main reason is because nodes delete bundles before they are transmitted - i.e., the average interval time is longer than the TTL of bundles.

Fig. 15 and 16 show the delivery ratio of all modified and un-modified protocols. We see that dynamic TTL has a higher delivery ratio than epidemic with constant TTL values. In particular, dynamic TTL significantly increases the delivery ratio over constant TTL by $40 \%$ in trace file and $20 \%$ in RWP model 


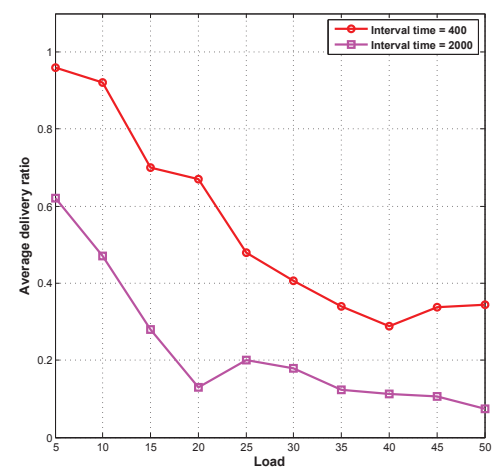

Fig. 14. Delivery ratio comparison of epidemic with TTL $=300$ in two scenarios with different interval times.

respectively. The higher delivery ratio is due to bundles having a dynamic TTL value corresponding to the intervals between encounters, which reduces the likelihood of nodes discarding bundles that have not arrived at their respective destination. Apart from that, with dynamic TTL, nodes also can effectively discard bundles after those bundles are exchanged. This is because the TTL set for bundles changed according to nodes encounters, therefore, once bundles are exchanged, old bundles are discarded from buffers in a short time, and nodes have more buffer size for new undelivered bundles.

We found that epidemic with EC+TTL increases delivery ratio and has over $80 \%$ delivery ratio in both RWP and trace file scenarios. In particular, in trace file experiments, epidemic with EC+TTL has a much higher delivery ratio than epidemic with EC when the load is 30 . For example, when the load is 45 , epidemic with EC+TTL results in more than $85 \%$ delivery ratio, whilst epidemic with EC has less than $60 \%$ delivery ratio. The reason for the higher delivery ratio is due to, on one hand, by the EC threshold of bundles, which encourages bundles duplication and hence, increases their delivery probability. On the other hand, with the use of TTL, nodes are able to free up their buffer and thus, store more undelivered bundles. Lastly, the delivery ratio of epidemic with cumulative immunity is similar to epidemic with immunity. This is because cumulative immunity is a buffer policy. It has no influence on the transmission of bundles before they are received by their respective destination.

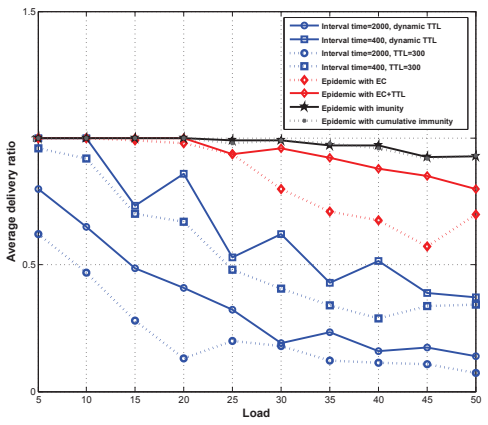

Fig. 15. Delivery ratio comparison of modified and un-modified protocols in RWP model.

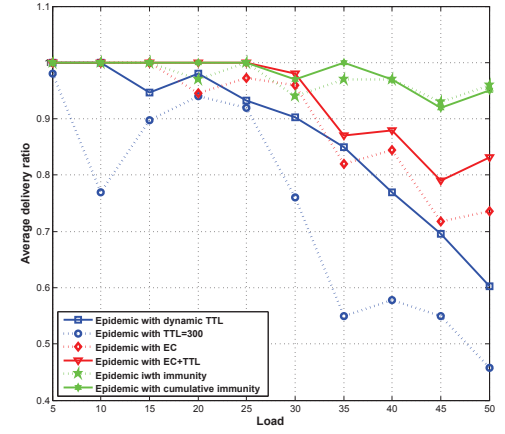

Fig. 16. Delivery ratio comparison of modified and un-modified protocols in trace-file based study.

2) Buffer Occupancy Level: Fig. 17 and 18 illustrate the buffer occupancy level of all modified protocols. We can observe that epidemic with dynamic TTL increases buffer occupancy level, but remains less than $20 \%$. The main reason is because dynamic TTL values lead to longer buffering time, and consequently, higher buffer occupancy level. This can be seen from Fig.17, in which the highest buffer occupancy level is reached in the scenario with 2000 seconds interval time. When the interval time is reduced, the buffer occupancy level decreases accordingly.

By comparing EC+TTL and epidemic with EC, we conclude that epidemic with EC+TTL reduces the buffer occupancy level of nodes. In the RWP model, the buffer occupancy level of epidemic with EC+TTL is $10 \%$ less than that of epidemic with EC only. However, given that nodes have fewer encounters in the trace file, the EC threshold is never reached, and hence, the TTL of bundles remains constant. As a result, nodes have a higher buffer occupancy level than in the RWP model. For example, when the load is at 20, epidemic with EC+TTL costs nodes less than $50 \%$ of their buffer in the RWP model, but is over $60 \%$ in trace file based experiments. Finally, the results highlight the effectiveness of using cumulative immunity tables in reducing nodes' buffer occupancy levels - as shown in RWP and trace file scenarios.

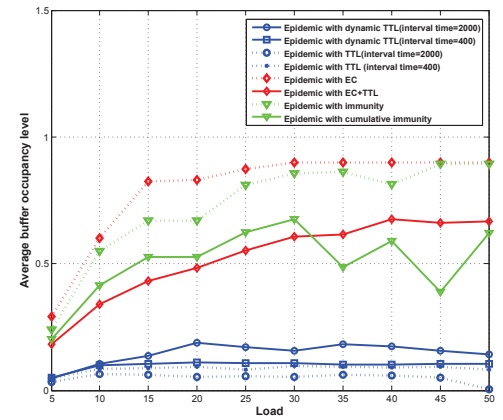

Fig. 17. Buffer occupancy level comparison of modified and un-modified protocols in RWP model.

3) Duplication Rate: Fig. 19 and 20 show that dynamic TTL has a different performance in terms of duplication rate. In the RWP model, dynamic TTL has a maximum $10 \%$ higher duplication rate as compared to experiments where nodes 


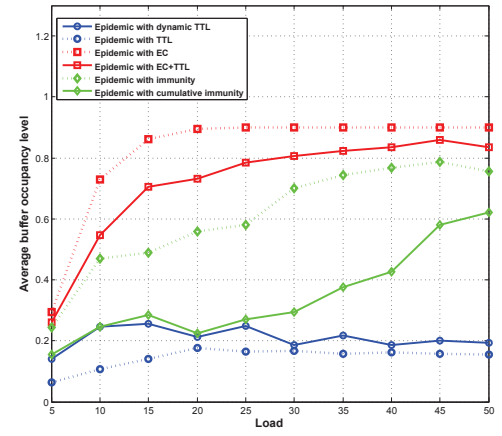

Fig. 18. Buffer occupancy level comparison of modified and un-modified protocols in trace-based study.

use a constant TTL. In trace file experiments, the maximum difference in duplication rate between dynamic and constant TTL is $20 \%$. The increased duplication rate is due to bundles having a lower probability of being discarded, and this is attributed to dynamic TTL values that let nodes store bundles until they encounter other nodes. As a result, bundles have a higher chance of being transmitted to more nodes, which increases duplication rate.

The results shown in both Fig. 19 and 20 also imply that epidemic with EC+TTL has a similar bundle duplication rate, in which the difference is less than $10 \%$. Note that, in both RWP and trace file experiments, when the load is greater than 30, epidemic with EC+TTL has a higher bundle duplication rate. This is because, unlike epidemic with EC, epidemic with EC+TTL sets a transmission count threshold values for each bundle. That is, before each bundle is deleted, it must have been transmitted a given number of times. As a result, bundles are dispatched to more nodes. On the other hand, when the load is less than 30, as the total number of bundles is small, the destination is able to receive all bundles in a short period of time. Consequently, there are fewer redundant bundles, and hence, two protocols have similar bundle duplication rate.

We see that the use of cumulative immunity tables reduces bundle duplication rate in both RWP and trace file experiments. Note, the bundle duplication rate is lower in the RWP model. This is because bundle duplication rate is closely related to the dissemination of the immunity table. In the trace file scenario, nodes have fewer encounters than those that move according to the RWP model. As a result, the immunity table is propagated more slowly to nodes, which leads to higher bundle duplication rate.

\section{Discussion}

In Table II, we compare original epidemic-based protocols and their enhanced counterparts. Note, all the values in the table are average values. First, we see that, except epidemic with immunity and cumulative immunity, enhanced protocols have a higher average delivery ratio. In particular, the biggest difference is between dynamic and constant TTL, where dynamic TTL improves delivery ratio in both tracefile and RWP experiments by $12 \%$ and $40 \%$ respectively. The

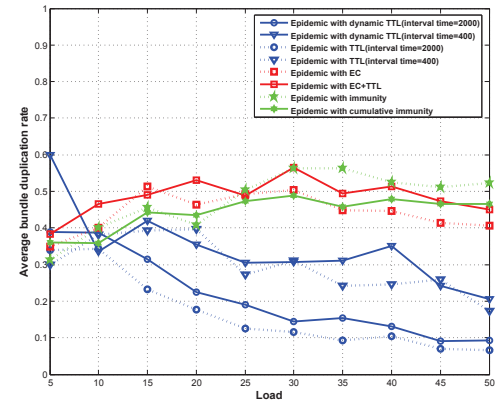

Fig. 19. Bundle duplication rate comparison of modified and un-modified protocols in RWP model.

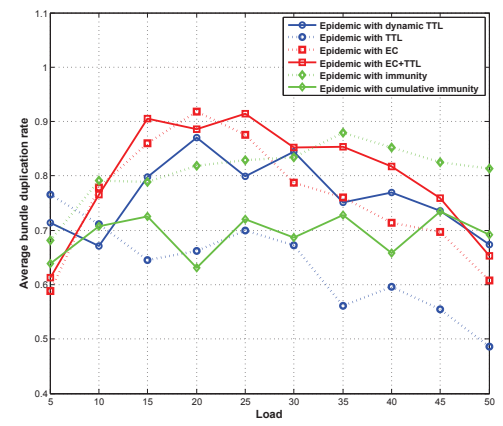

Fig. 20. Bundle duplication rate comparison of modified and un-modified protocols in trace-file study.

main reason for this significant improvement is that nodes are able to adapt the TTL of bundles in accordance with varying contact duration. Similarly, epidemic with EC+TTL avoids discarding bundles prematurely. Both epidemic with immunity and cumulative immunity have the same delivery ratio. However, as we will discuss later, they have a low buffer occupancy level.

Second, dynamic TTL has a higher buffer occupancy level - in fact the lowest recorded buffer occupancy level is $12 \%$ and $11 \%$ higher than its original counterpart in trace-file and RWP experiments respectively. This, however, leads to superior bundle delivery ratio as bundles are stored by nodes for a longer period time, especially when the frequency of contact is low. From our experiments, we find that epidemic with EC and EC+TTL have the highest buffer occupancy level. However, by incorporating a TTL value, the buffer occupancy level reduces from $79 \%$ to $57 \%$ and from $74 \%$ to $59.5 \%$ in trace file and RWP studies respectively. In other words, EC+TTL has approximately $20 \%$ lower buffer occupancy level than its counterpart.

Third, except for epidemic with cumulative immunity, our enhancements have slightly higher bundle duplication rate. In particular, dynamic TTL increases the lowest duplication rate from $66 \%$ to $69 \%$ and from $13.8 \%$ to $22.8 \%$ respectively in trace file and RWP experiments. Note that, epidemic with immunity has the highest duplication rate $-82 \%$ in trace file, and $48 \%$ in RWP experiments. Advantageously, a high bundle duplication rate means better delivery ratio - as demonstrated 
TABLE II

COMPARISON OF ORIGINAL AND ENHANCED PROTOCOLS.

\begin{tabular}{|l|c|c|c|c|c|c|}
\hline \multirow{2}{*}{} & \multicolumn{2}{|c|}{$\begin{array}{c}\text { Delivery rate } \\
(\%)\end{array}$} & \multicolumn{2}{c|}{$\begin{array}{c}\text { Buffer occupancy } \\
\text { level (\%) }\end{array}$} & \multicolumn{2}{c|}{$\begin{array}{c}\text { Duplication Rate } \\
\text { (\%) }\end{array}$} \\
\cline { 2 - 7 } & RWP & Trace file & RWP & Trace file & RWP & Trace file \\
\hline $\begin{array}{l}\text { Epidemic with } \\
\text { TTL }\end{array}$ & 24.6 & 74.4 & 5.1 & 11.3 & 13.8 & 66.3 \\
\hline $\begin{array}{l}\text { Epidemic with } \\
\text { Dyanmic TTL }\end{array}$ & 64.7 & 86.8 & 16.3 & 23.3 & 22.8 & 75.4 \\
\hline $\begin{array}{l}\text { Epidemic with } \\
\text { EC }\end{array}$ & 76.4 & 88.2 & 74.6 & 79.7 & 45.7 & 79.2 \\
\hline $\begin{array}{l}\text { Epidemic with } \\
\text { EC+TTL }\end{array}$ & 92.5 & 93.6 & 59.5 & 57.1 & 49.3 & 80.3 \\
\hline $\begin{array}{l}\text { Epidemic with } \\
\text { Immunity table }\end{array}$ & 97.7 & 95.3 & 72.5 & 58.2 & 48.5 & 82.4 \\
\hline $\begin{array}{l}\text { Epidemic with } \\
\begin{array}{l}\text { Cumulative Immunity } \\
\text { table }\end{array}\end{array}$ & 98.4 & 98.6 & 45.8 & 32.8 & 35.5 & 69.4 \\
\hline
\end{tabular}

by epidemic with dynamic TTL and EC+TTL. This is a fundamental feature of epidemic-based protocols as they are highly dependent on contact frequency. This means when bundle duplication rate is high, bundles can be forwarded quickly throughout the network, and thereby, lead to high bundle delivery ratios. Apart from that, our results show that epidemic with a cumulative immunity table is able to maintain a high delivery ratio with low duplication rate. This is primarily due to the effectiveness of the cumulative immunity table in purging received bundles from nodes.

\section{CONCLUSION}

In this paper, we have compared epidemic-based protocols using a unified framework. Specifically, we used the same mobility models, i.e., trace-file and RWP, and performance metrics to compare key epidemic-based protocols. We found that P-Q epidemic increases transmission delay and has poor bundle delivery ratio. Epidemic with immunity table has the highest delivery ratio at the expense of higher buffer occupancy level. In addition, the use of a constant TTL value results in poor performance as nodes in DTNs have wide ranging contact intervals. In addition, epidemic with EC experiences high buffer occupancy level and long delivery delay. Accordingly, we propose three enhancements to address these limitations: epidemic with dynamic TTL, EC+TTL and cumulative immunity table. Our extensive experiments show these enhancements to have high delivery ratio. Moreover, the use of cumulative immunity tables helps reduce duplication rate and buffer occupancy level significantly.

\section{REFERENCES}

[1] P. Juang, H. Oki, Y. Wang, M. Martonosi, L. Peh, and D. Rubenstein, "Energy-efficient computing for wildlife tracking: Design tradeoffs and early experiences with zebranet," ACM SIGPLAN NOTICES, vol. 37, no. 10, pp. 96-107, October 2002.

[2] BBC, "SA pigeon faster than broadband," http://news.bbc.co.uk/2/hi/africa/8248056.stm, 2009.

[3] N. Glance, D. Snowdon, and J.-L. Meunier, "Pollen: using people as a communication medium," Computer Networks, vol. 35, no. 4, pp. 429 $-442,2001$.

[4] P. Hui, A. Chaintreau, J. Scott, R. Gass, J. Crowcroft, and C. Diot, "Pocket switched networks and human mobility in conference environments," in Proceedings of ACM SIGCOMM Workshops, Philadelphia, PA, USA, August 22-26 2005.
[5] D. Geraghty, "Sensor networking with delay tolerance (SeNDT)," http://down.dsg.cs.tcd.ie/sendt, 2006.

[6] U. Lee, E. Magistretti, B. Zhou, M. Gerla, P. Bellavista, and A. Corradi, "Mobeyes: Smartmobs for urban monitoring with a vehicular sensor network," IEEE Wireless Communications, vol. 13(5), pp. 52-57, (2006).

[7] V. Lenders, G. Karlsson, and M. May, "Wireless ad hoc podcasting," in IEEE SECON, San Diego, California, USA, June 18-21 2007, pp. 273-283.

[8] X. Zhang, G. Neglia, J. Kurose, and D. Towsley, "Performance modeling of epidemic routing," Computer Networks, vol. 51, no. 10, pp. 2867 2891, July 2007.

[9] F. Bai, N. Sadagopan, and A. Helmy, "IMPORTANT: a framework to systematically analyze the impact of mobility on performance of routing protocols for adhoc networks," in IEEE INFOCOM, San Francisco, CA, USA, March 2003.

[10] T. Matsuda and T. Takine, "(p,q)-epidemic routing for sparsely populated mobile ad hoc networks," IEEE JSAC, vol. 26, no. 5, pp. 783-793, June 2008.

[11] Y. Hayel and H. Tembine, "Information dissemination using epidemic routing with delayed feedback," in Proceedings of IEEE Internatonal Conference on Mobile Adhoc and Sensor Systems, Pisa, Italy, October 8-11 2007, pp. 1-4.

[12] P. Mundur, M. Seligman, and J. N. Lee, "Immunity-based epidemic routing in intermittent networks," in IEEE SECON, California, USA, June 16-20 2008, pp. 609-611.

[13] A. Vahdat and D. Becker, "Epidemic routing for partially-connected ad hoc networks," in Technologies Report CS. Department of Computer Science, Duke University, 2002.

[14] J. Davis, A. Fagg, and B. Levine, "Wearable computers as packet transport mechanisms in highly-partitioned ad-hoc networks," in Proceedings of Fifth International Symposium on Wearable Computers(ISWC), Zurich, Switzerland, October 18-21 2001, pp. 141-148.

[15] K. A. Harras, K. C. Almeroth, and E. M. Belding-Royer, Delay Tolerant Mobile Networks (DTMNs): Controlled Flooding in Sparse Mobile Networks. Springer Berlin / Heidelberg, 2005, ch. Lecture Notes in Computer Science, pp. 1180-1192.

[16] J. Scott, R. Gass, J. Crowcroft, P. Hui, C. Diot, and A. Chaintreau, "CRAWDAD trace cambridge/haggle/imote/intel (v. 2006-01-31)," Downloaded from http://crawdad.cs.dartmouth.edu/cambridge/haggle/imote/intel, Jan. 2006.

[17] D. Kotz, T. Henderson, and I. Abyzov, "CRAWDAD data set dartmouth/campus (v. 2004-12-18)," Downloaded from http://www.crawdad.org/dartmouth/campus, Dec. 2004.

[18] M. Zarafshan-Araki and K.-W. Chin, "TrainNet: A transport system for delivering non real-time data," Computer Communications, vol. 33, no. 15 , pp. 1850 - 1863, jan 2010.

[19] G. Resta and P. Santi, "An analysis of the node spatial distribution of the random waypoint model for ad hoc networks," in ACM Workshop on Principles of Mobile Computing (POMC), Toulouse, France, July 2007. 